\title{
False aneurysm with median nerve palsy after iatrogenic brachial artery puncture
}

\author{
KMH Yip, H Yurianto, J Lin
}

\begin{abstract}
Summary
We report on a case in which a patient on oral anticoagulation for her aortic valve replacement, with an International Normalised Ratio of 2.13, developed a false aneurysm of the brachial artery after a routine arterial puncture, despite direct pressure to the aspiration site. The false aneurysm was complicated by the development of median nerve palsy.
\end{abstract}

Keywords: false aneurysm, arterial puncture

Complications after arterial puncture are extremely low, despite the frequency with which this procedure is performed. The complication of false aneurysm is even more rare and usually occurs in drug addicts and in patients on haemodialysis.

\section{Case report}

A 64-year-old woman was admitted to a medical ward complaining of palpitations and shortness of breath. She had a history of mixed aortic valve disease and had undergone aortic valve replacement in 1985 . As the prosthesis was a metallic valve, she was placed on longterm oral anticoagulation, consisting of warfarin $2 \mathrm{mg}$ daily. At the time of admission, she was also noted to be suffering from mitral valve stenosis with atrial fibrillation and chronic obstructive airways disease.

During the routine blood investigations, attempts to obtain an arterial blood sample from both radial arteries for arterial blood gases had failed, and therefore arterial blood was taken from the right brachial artery. The patient was then told to compress the sampling point for five minutes. One hour later, she noticed a painless swelling over the right cubital fossa (figure). She also noticed bruising over her forearm extending to the dorsum of her hand. The following day, she also noticed numbness and tingling over the lateral aspect of her right hand, but motor function was normal. Routine blood investigations showed that the clotting was prolonged with an International Normalised Ratio (INR) of 2.13.

The patient was referred to the orthopaedic team for further management. Physical examination revealed a nonpulsatile solid mass with ill-defined borders over the centre of her right cubital fossa measuring $6 \mathrm{~cm}$ in diameter. A pulsatile vessel was clearly visible on the medial side of the mass. The radial pulse was normal.
There was loss of sensation over the median nerve distribution but no motor deficit.

Ultrasound examination of the mass revealed a pseudo-aneurysm of the right brachial artery measuring $4.7 \times 3.8 \times 3.3 \mathrm{~cm}$. The patient underwent surgical exploration without a tourniquet. A curved medial incision was made and after exposing the pseudo-aneurysm the mass was found to arise from the right brachial artery. Deep dissection revealed that the aneurysm was compressing the median nerve. Having obtained vascular control, the mass was excised and the defect was repaired by direct suture. Postoperatively the patient made an uneventful recovery and the neurological symptoms resolved after two days.

\section{Discussion}

A false aneurysm is described as a pulsatile haematoma occurring as a result of disruption of a portion of the arterial wall. These may

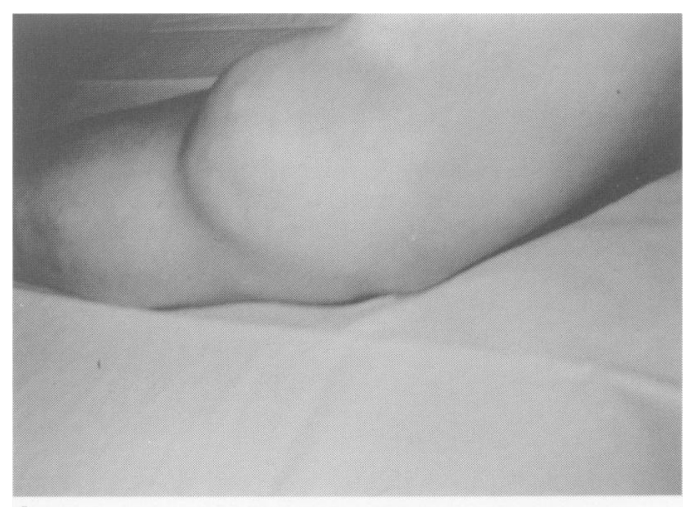

A

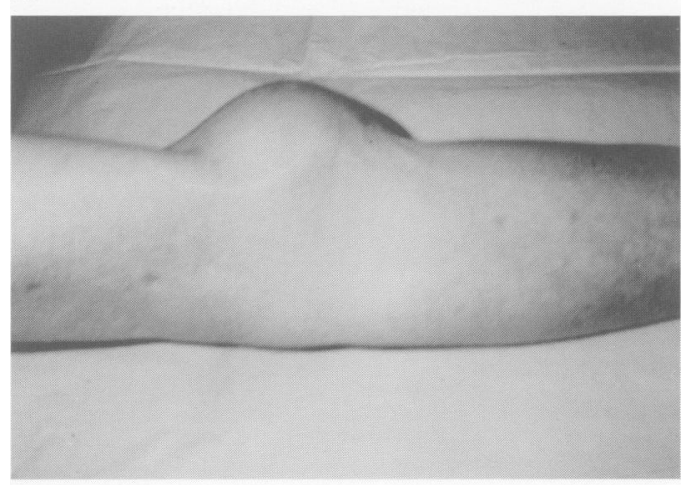

Figure Frontal (A) and lateral (B) views of the false aneurysm of the brachial artery at the right cubital fossa 
occur after blunt or penetrating arterial injuries, repetitive punctures and traction injury to the artery. False aneurysm after arterial puncture is rare. It was thought to be due to faulty technique ${ }^{1}$ or due to abnormal coagulation. ${ }^{2}$ Such a complication is more commonly seen in drug addicts ${ }^{3,4}$ and in patients on haemodialysis. ${ }^{5,6}$ If the mass is large, there may be an associated median nerve palsy as a result of compression.

The common symptoms of an aneurysm occurring in the upper limb are that of a painful pulsatile mass with associated tenderness. Although a mass is almost always present, tension in the subfascial layer and brawny oedema of the skin may mask the pulsatility. There may be symptoms associated with distal embolisation or local nerve compression. ${ }^{2}$ Coolness, pallor, parasthesia, or numbness distal to the lesion may be present as a result of the embolisation. A bruit may be present in some cases.

Investigations for pre-operative confirmation of the aneurysm include ultrasound in order to detect the site of the leak. Arteriogram is not always absolutely necessary but may be useful as a guide during the operation. Attempted drainage or biopsy may have undesirable consequences and should not be attempted.

1 Rapport S, Sniderman KW, Morse SS, Proto MH, Ross GR. Pseudoaneurysm: a complication of faulty technique in femoral arterial puncture. Radiology 1985; 154: 529-30.

2 Littler WA. Median nerve palsy. A complication of brachial artery cannulation. Postgrad Med $\mathcal{f} 1976 ; 52: 110-3$.

3 Ortery cannulation. Postgrad Med $\mathcal{F} 1976 ; 52: 110-3$. with brachial plexus palsy: a complication of parenteral drug addiction. Am $\mathcal{F}$ Emerg Med 1990; 8: 129-33.
If the median nerve is compressed, early surgical intervention is indicated.

In clinical practice, the radial artery is the most common site of arterial puncture. One of the reasons is its superficial locality, and the other is that if the artery were to become thrombosed or otherwise compromised, then the hand is still able to receive its arterial blood supply via the ulnar artery. More central vessels such as the brachial are not so favoured as it is the main artery supplying the distal forearm and hand. However, if it is difficult to puncture distal vessels then more proximal ones are utilised but due care must be taken to ensure good haemostasis after puncture.

In this case report, this patient had an INR of 2.13 at the time of admission and hence an appropriate dose was being taken. Nevertheless, in any patient on oral anticoagulation, precautions should be taken to ensure haemostasis after arterial puncture. Although the patient was compliant and applied pressure to the area as requested, she may have applied pressure to an inappropriate site, therefore a staff member should have been enlisted to ensure good control. This case is a useful reminder that even simple arterial puncture can lead to relatively serious complications if appropriate precautions are not taken.

4 Benitez PR, Newell MA. Vascular trauma in drug abuse: pattern of injury. Ann Vasc Surg 1986; 1: 175-81.

5 Wattern of injury. Ann Vasc Surg 1986; 1: 175-81. Weiner SN. Complications of vascular acces
hemodialysis. Angiology 1985; 36: 275-84.

6 Fransson SG, Thomsen MB. Arterial pseudoaneurysm in a haemodialysis patient. Vasa 1983; 137: 1173. 